\title{
Predicción de la distribución de dividendos en Argentina empleando un modelo lineal generalizado mixto
}

Prediction on the distribution of dividends in Argentina using a mixed generalized linear model

\author{
Mariana Verónica González \\ Facultad de Ciencias Económicas, \\ Universidad Nacional de Córdoba, \\ Argentina. \\ E-mail:mvgonzalez@eco.unc.edu.ar
}

\author{
Adrián Maximiliano \\ Moneta Pizarro \\ Facultad de Ciencias Económicas, \\ Universidad Nacional de Córdoba, Argentina. \\ E-mail: amoneta@eco.uncor.edu
}

Fecha de recepción: 17/03/2016 Fecha de aprobación: 22/08/2016

\section{Resumen}

Durante las últimas décadas, la política de dividendos seguida por las empresas ha sido un tema de interés en diversas investigaciones, con apreciaciones distintas sobre los resultados conseguidos. En general, se admite que la decisión de distribuir dividendos por parte de una empresa es el resultado de un conjunto de factores relacionados, desde limitaciones de carácter jurídico hasta cuestiones vinculadas a la estructura financiera de la entidad y su situación de liquidez, pasando por la capacidad de la empresa para generar beneficios de manera sostenida y las necesidades de fondos impuestas por sus proyectos de inversión. En este sentido, muchos autores utilizan un conjunto de ratios contables para medir la sensibilidad de dichas variables frente a la decisión de repartir cantidades a cuenta de beneficios.

En este trabajo se ajustó un modelo logístico mixto para predecir la decisión de distribuir dividendos por parte de empresas argentinas que cotizaron en bolsa, entre los años 2003 y 2010, utilizando información

Palabras clave

- dividendos

- modelo logístico mixto

- rentabilidad

- Argentina de los estados contables y ratios definidos en la bibliografía. Se destaca el importante poder predictivo del indicador que mide la rentabilidad en función de las ganancias de la explotación, definido como el cociente entre la utilidad antes de intereses e impuestos y el activo total de la empresa. 


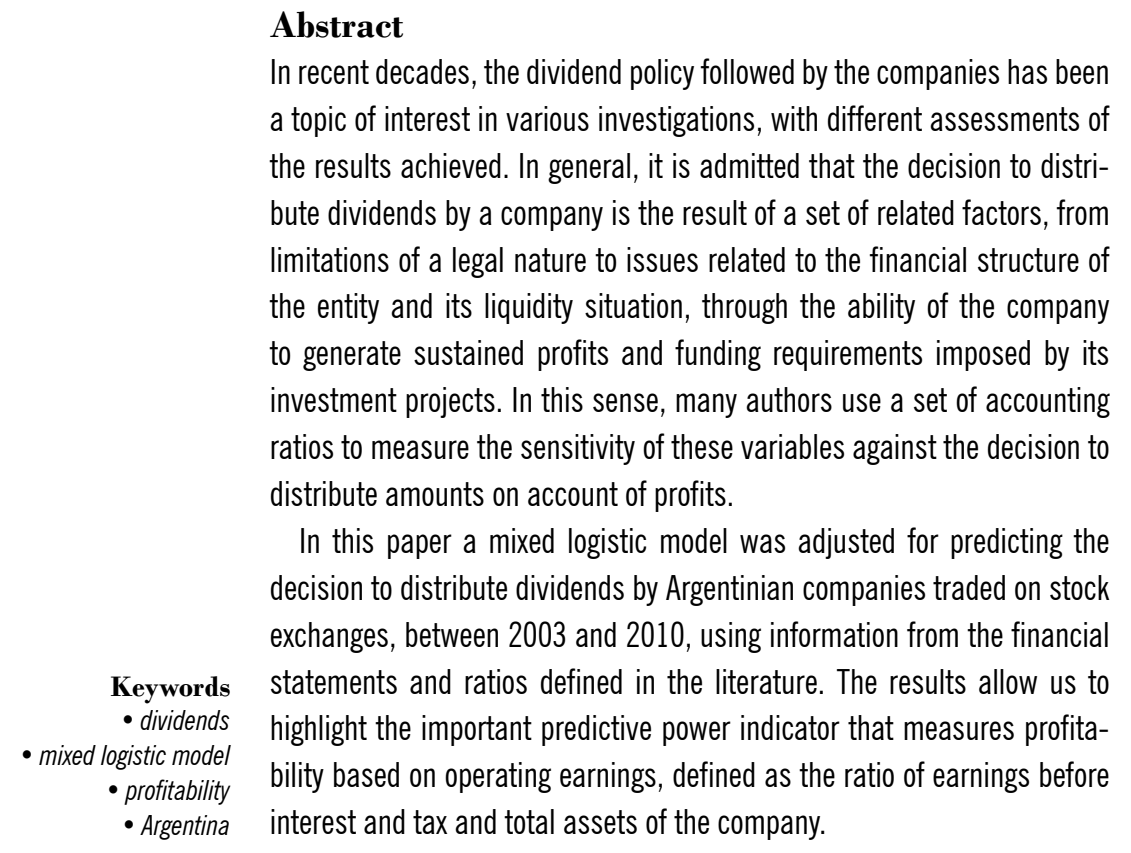

\section{Introducción}

La política de dividendos adoptada por las empresas es y ha sido un tema de interés en diversas investigaciones, con diferentes enfoques y perspectivas. Las líneas de investigación encaminadas a solucionar esta cuestión están concebidas desde dos puntos de vista diferentes: por un lado, medir las repercusiones de la estrategia del reparto de dividendos en los precios de las acciones, y, por otro, conocer los factores que influyen en la decisión de repartir beneficios. Los esfuerzos orientados a dar respuesta a la segunda cuestión se han ocupado, principalmente, del desarrollo de modelos de comportamiento de los dividendos que, a partir de variables macro 0 micro financieras, permitieran explicar y predecir las políticas aplicadas por las empresas.

En general, se admite que la decisión de distribuir dividendos por parte de una empresa es el resultado de un conjunto de factores relacionados, desde limitaciones de carácter jurídico hasta cuestiones vinculadas a la estructura financiera de la entidad y su situación de liquidez, pasando por la capacidad de la empresa para generar beneficios de manera sostenida y las necesidades de fondos impuestas por sus proyectos de inversión. En este sentido, muchos autores utilizan un conjunto de ratios contables para medir la sensibilidad de dichas variables frente a la decisión de repartir cantidades a cuenta de beneficios.

En función de lo expuesto anteriormente, este estudio tiene como objetivo general analizar si el comportamiento de determinados indicadores contables, calculados con la información publicada periódicamente por las empresas que cotizan en bolsa, influye en la decisión de distribuir dividendos. Para ello se propone un modelo logístico mixto, en el que se introduce una ordenada al origen aleatoria para captar el efecto sujeto-específico (Fahrmeir y Tutz, 2001; Diggle et ál., 2002). Se trabaja con empresas de Argentina que cotizaron en la Bolsa de Comercio de Buenos Aires, entre los años 2003 y 
2010, utilizando información de los estados contables y ratios definidos en la bibliografía.

El resto del trabajo se organiza en cinco secciones además de esta introducción. La segunda sección ofrece una revisión de la literatura sobre distribución de dividendos y los instrumentos estadísticos aplicados para predecirla. La tercera sección se enfoca en la metodología, describiendo no sólo el modelo empleado sino también el mecanismo de selección de la muestra y las covariables consideradas. La cuarta sección presenta los resultados y la última sección ofrece las conclusiones del trabajo.

\section{Antecedentes}

La cuantía y la estabilidad de los dividendos son los dos aspectos de la política de dividendos, aunque no los únicos, que mayor atención han merecido en la literatura financiera. Si bien el indicador de mayor valor informativo, en relación con la rentabilidad de una empresa, debiera ser el beneficio operativo neto, por razones psicológicas y de preferencia por la liquidez, el dividendo parece ser, para el accionista, el mejor indicador de la situación económicofinanciera de la empresa, por ser algo tangible que genera confianza en la gestión y se traduce en una cotización relativamente estable de las acciones de la empresa en el mercado (Suárez Suárez, 1986).

Un aspecto relevante, y sin duda vinculado al anterior, es el efecto de la decisión de dividendos sobre el valor de la empresa en el mercado y, más precisamente, la discusión en torno a si el precio de las acciones es independiente 0 no de la política de dividendos (Miller y Modigliani, 1961; Friend y Puckett, 1964; Gordon, 1962).

En este contexto, una cuestión clave se resume en el siguiente interrogante: ipor qué las empresas distribuyen dividendos? Podría pensarse que una motivación es satisfacer a los accionistas actuales y atraer otros nuevos. Sin embargo, la retención de beneficios puede considerarse como un factor indicativo de la capacidad de empresa para generar oportunidades rentables de inversión y los dividendos no percibidos por los accionistas en el momento actual podrían verse compensados con futuras ganancias de capital. La respuesta a esta cuestión, por lo tanto, no es simple y responde a una multiplicidad de factores (Black, 1976).

La discusión en torno a la relevancia o no de la política de dividendos se viene desarrollando en el seno de la economía financiera desde comienzo de los años sesenta y ha dado lugar a distintas teorías sobre el papel de los dividendos en el proceso de valoración de la empresa. La investigación empírica sobre los efectos de la política de dividendos se ha desarrollado junto con la teoría, y no existe tampoco un consenso sobre la cuestión. Algunos autores han interpretado las oscilaciones en la cotización de las acciones como efecto directo de la política de dividendos. Sin embargo, la evidencia de este fenómeno tampoco es clara, ya que las estimaciones efectuadas no permiten distinguir entre variaciones en las cotizaciones originadas por la información transmitida al mercado a través de las oscilaciones del dividendo y variaciones en las cotizaciones por efecto puro del dividendo (Jensen y Smith, 1984). Sin embargo, las posturas enfrentadas coinciden en señalar que los dividendos transmiten información al mercado bursátil acerca de las expectativas futuras de la empresa.

Las investigaciones empíricas se centran en determinar qué factores influyen en la distribución de dividendos; se puede citar como pioneros los estudios de Lintner (1956), Darling (1957), Dhrymes y Kurz (1964) y Fama y Babiak (1968). Todos estos autores analizan la correlación existente entre los dividendos y una serie de variables económicofinancieras como el nivel de beneficios y su estabilidad 0 crecimiento, la inversión, la dimensión de la empresa, la liquidez, el endeudamiento, etc. Entre los estudios más recientes se destacan los de Truong y Heaney (2002), Kanwer (2003), Asadi y Azizi Basir (2008) y Tabari y Shirazi (2015). En la Tabla $N^{0} 1$ se sintetizan las principales características de estas investigaciones. 
Tabla 1. Algunas investigaciones empíricas

\begin{tabular}{|c|c|c|}
\hline AUTOR/ES & METODOLOGÍA & RESULTADOS \\
\hline $\begin{array}{l}\text { Lintner } \\
(1956)\end{array}$ & $\begin{array}{l}\text { Modelo econométrico basado en un } \\
\text { conjunto de entrevistas con gerentes } \\
\text { de empresas. }\end{array}$ & $\begin{array}{l}\text { La decisión sobre el dividendo está íntimamente conecta- } \\
\text { da con su importe en el ejercicio anterior y con el volumen } \\
\text { de las ganancias netas (después de impuestos). }\end{array}$ \\
\hline $\begin{array}{l}\text { Darling } \\
\text { (1957) }\end{array}$ & $\begin{array}{l}\text { Modifica el modelo de Lintner } \\
\text { y sugiere una explicación más } \\
\text { completa del comportamiento de los } \\
\text { dividendos, incluyendo factores como } \\
\text { la liquidez y las expectativas. }\end{array}$ & $\begin{array}{l}\text { Concluye que, para el colectivo de las grandes empresas } \\
\text { industriales, los dividendos tienden a variar directamente } \\
\text { con las ganancias netas del presente ejercicio y del } \\
\text { anterior y las amortizaciones. Por el contrario, lo hacen en } \\
\text { relación inversa, con el volumen de las ventas. }\end{array}$ \\
\hline $\begin{array}{l}\text { Dhrymes y Kurz } \\
\text { (1964) }\end{array}$ & $\begin{array}{l}\text { Amplio trabajo econométrico acerca } \\
\text { de las empresas de energía eléctrica. } \\
\text { Incorpora variables no consideradas } \\
\text { por Lintner. }\end{array}$ & $\begin{array}{l}\text { El pago de dividendos está notoriamente afectado por } \\
\text { variables no consideradas en los modelos anteriores, } \\
\text { como el programa de inversiones de la empresa, el nivel } \\
\text { de endeudamiento a largo plazo, tamaño de la firma y } \\
\text { status de control (dominada o no). }\end{array}$ \\
\hline $\begin{array}{l}\text { Fama y Babiak } \\
\text { (1968) }\end{array}$ & $\begin{array}{l}\text { Comparan el modelo de Lintner con } \\
\text { otros alternativos, empleando una } \\
\text { técnica de simulación para estudiar } \\
\text { las propiedades de los parámetros. }\end{array}$ & $\begin{array}{l}\text { De las especificaciones contrastadas, las dos más } \\
\text { adecuadas son las de Lintner y otra que se comporta algo } \\
\text { mejor, donde figuran las ganancias de la empresa, como } \\
\text { variable retardada. }\end{array}$ \\
\hline $\begin{array}{l}\text { Truong y Heaney } \\
\text { (2002) }\end{array}$ & $\begin{array}{l}\text { Estudiaron la política de dividendos } \\
\text { en diferentes países, en base a una } \\
\text { muestra de } 37 \text { empresas. }\end{array}$ & $\begin{array}{l}\text { La probabilidad que las empresas paguen dividendos } \\
\text { es mayor cuando tienen una alta rentabilidad, oportuni- } \\
\text { dades de inversión limitadas y mayoría de accionistas } \\
\text { extranjeros. }\end{array}$ \\
\hline Kanwer (2003) & $\begin{array}{l}\text { Se recogieron datos de } 317 \text { empresas } \\
\text { que cotizan en la Bolsa de Valores de } \\
\text { Pakistán y se analizaron mediante } \\
\text { regresión logística. }\end{array}$ & $\begin{array}{l}\text { Los resultados muestran que existe una relación negativa } \\
\text { significativa entre los resultados no asignados cada año } \\
\text { y los dividendos. Por otra parte, las oportunidades de } \\
\text { inversión disponibles tienen un impacto significativo en la } \\
\text { política de dividendos de la compañía. }\end{array}$ \\
\hline $\begin{array}{l}\text { Asadi y Azizi } \\
\text { Basir (2008) }\end{array}$ & $\begin{array}{l}\text { Examinaron mediante un modelo de } \\
\text { regresión la relación entre la ren- } \\
\text { tabilidad y la liquidez y su impacto } \\
\text { sobre los dividendos 1997-2003. La } \\
\text { muestra estaba compuesta por } 49 \\
\text { empresas que cotizan en la Bolsa de } \\
\text { Teherán. }\end{array}$ & $\begin{array}{l}\text { Los resultados sugieren que existe una fuerte relación } \\
\text { entre la rentabilidad y la distribución de dividendos. № } \\
\text { obstante, existe una débil relación entre la liquidez y la } \\
\text { política de dividendos. }\end{array}$ \\
\hline $\begin{array}{l}\text { Tabari y Shirazi } \\
\text { (2015) }\end{array}$ & $\begin{array}{l}\text { Trabajaron con } 109 \text { empresas para } \\
\text { el período comprendido entre } 2008 \\
\text { y 2012. Aplicaron una regresión de } \\
\text { panel con efectos fijos. }\end{array}$ & $\begin{array}{l}\text { Encontraron una relación positiva significativa entre el } \\
\text { activo, la liquidez, las oportunidades de crecimiento tangi- } \\
\text { bles, el tamaño de la empresa y el pago de dividendos. }\end{array}$ \\
\hline
\end{tabular}


Otros trabajos basan su investigación en la información contable emanada de los estados contables. Entre los autores que encontraron que los ratios contables son una fuente apropiada de información para explicar el pago de dividendos podemos citar a Rodríguez Fernández (1988), Rodríguez Enríquez (2003), Guzmán (2004) y García Borbolla y Larrán Jorge (2005). El trabajo de Rodríguez Fernández (1988) resulta interesante porque es uno de los primeros que emplea un modelo de elección discreta (logit) para estudiar los determinantes de la decisión de distribuir dividendos, considerando inicialmente 20 ratios contables. Rodríguez Enríquez (2003) realiza, antes de aplicar la regresión logística, el Test de Mann-Whitney para cada uno de los 34 ratios contables seleccionados, a fin de analizar su capacidad para separar las empresas que reparten dividendos de las que no lo hacen. Un procedimiento similar emplean García Borbolla y Larrán Jorge (2005), con la novedad que incorporan como variable explicativa los dividendos repartidos en el ejercicio anterior. El estudio de Guzmán (2004) también se realizó en dos fases: primeramente se aplicó una reducción de variables a través del análisis de componentes principales para conocer los factores subyacentes existentes entre los 10 ratios seleccionados y, en una segunda etapa, a partir de las puntualizaciones factoriales obtenidas, se aplicó una regresión logit sobre la variable dicotómica establecida, según las empresas muestreadas hubiesen 0 no repartido dividendos en el ejercicio analizado. La Tabla № 2 refleja una síntesis de los trabajos que emplean ratios contables.
Respecto de los instrumentos estadísticos aplicados, puede observarse que muchos trabajos coinciden en la utilización del análisis de regresión para la estimación del poder explicativo de diferentes variables. Otros aplican técnicas multivariadas como el análisis de componentes principales o la regresión logística, además de las herramientas propias de la estadística descriptiva. En este trabajo se propone la aplicación de modelos estadísticos avanzados, conocidos como Modelos Lineales Generalizados Mixtos (MLGM), que, en forma general, permiten modelar conjuntos de datos en los que las observaciones no son independientes. La modelación de datos reales en el marco teórico de estos modelos brinda la posibilidad de analizar datos con estructuras de dependencia, desbalances y falta de normalidad. Es así posible relajar los supuestos tradicionales del modelo lineal general y modelar, de manera flexible, estructuras más complicadas de datos. Los modelos mixtos se adecúan bien a situaciones donde una variable se evalúa en varios momentos del tiempo y, por tanto, es razonable asumir que existen correlaciones entre observaciones dentro del mismo sujeto. La modelación en el marco de los modelos mixtos maneja estas correlaciones mediante la incorporación de variables aleatorias o mediante la modelación directa de la matriz de covarianzas residual. Existen muchos beneficios que pueden obtenerse con el uso de modelos mixtos. En algunas situaciones se incrementa la precisión de las estimaciones. En otras se contempla mejor la estructura y se amplía el espacio de inferencia, sobre todo cuando la estructura de los datos es jerárquica (Rabe-Hesketh y Skrondal, 2012). 
Tabla 2. Modelos que explican la decisión de distribuir dividendos empleando ratios contables

\begin{tabular}{|c|c|c|c|c|}
\hline AUTOR/ES & $\begin{array}{l}\text { MUESTRA Y PERÍODO } \\
\text { DE ANÁLISIS } \\
\end{array}$ & $\begin{array}{l}\text { RATIOS } \\
\text { SIGNIFICATIVOS } \\
\end{array}$ & METODOLOGÍA & RESULTADOS \\
\hline $\begin{array}{l}\text { Rodríguez } \\
\text { Fernández (1988) }\end{array}$ & $\begin{array}{l}98 \text { grandes empresas } \\
\text { españolas de diversas } \\
\text { ramas de actividad } \\
\text { económica. Ejercicio } \\
\text { del año } 1983\end{array}$ & $\begin{array}{l}\text { - Rentabilidad neta del } \\
\text { activo total } \\
\text { - Valor añadido / } \\
\text { ventas } \\
\text { - Equilibrio financiero } \\
\text { - Deudas a corto plazo } \\
\text { / ventas } \\
\text { - Capacidad de auto- } \\
\text { financiación / deuda a } \\
\text { corto plazo }\end{array}$ & $\begin{array}{l}\text { Modelo de elección } \\
\text { dicotómica, con } \\
\text { estimación máximo } \\
\text { verosímil }\end{array}$ & $\begin{array}{l}\text { Mayores valores de los } \\
\text { primeros cuatro ratios } \\
\text { implican una más } \\
\text { alta probabilidad de } \\
\text { distribuir dividendos. El } \\
\text { último ratio interviene } \\
\text { con un efecto diferen- } \\
\text { cial negativo }\end{array}$ \\
\hline $\begin{array}{l}\text { Rodríguez } \\
\text { Enríquez (2003) }\end{array}$ & $\begin{array}{l}\text { Empresas no financie- } \\
\text { ras de Asturias. Período } \\
\text { 1994-1996 }\end{array}$ & $\begin{array}{l}\text { - Endeudamiento: } \\
\text { Pasivo exigible / pasivo } \\
\text { total } \\
\text { - Rentabilidad econó- } \\
\text { mica: (beneficio neto } \\
\text { + gastos financieros) / } \\
\text { activo neto total }\end{array}$ & $\begin{array}{l}\text { - Test de Mann-Whit- } \\
\text { ney para cada ratio } \\
\text { - Regresión logística }\end{array}$ & $\begin{array}{l}\text { La rentabilidad eco- } \\
\text { nómica incrementa la } \\
\text { probabilidad de repartir } \\
\text { dividendos. Un mayor } \\
\text { endeudamiento reduce } \\
\text { las probabilidades de } \\
\text { reparto }\end{array}$ \\
\hline Guzmán (2004) & $\begin{array}{l}57 \text { sociedades no fi- } \\
\text { nancieras que cotizaron } \\
\text { en el mercado español } \\
\text { durante los ejercicios } \\
1999 \text { a } 2001\end{array}$ & $\begin{array}{l}\text { - Productividad: } \\
\text { (ingresos - gastos) / } \\
\text { gastos de personal. } \\
\text { - Resultados por } \\
\text { empleado: beneficios } \\
\text { brutos / cantidad de } \\
\text { empleados }\end{array}$ & $\begin{array}{l}\text { - Análisis de compo- } \\
\text { nentes principales } \\
\text { - Regresión logística }\end{array}$ & $\begin{array}{l}\text { Fuerte relación de cau- } \\
\text { salidad entre el compo- } \\
\text { nente de productividad y } \\
\text { el reparto de dividendos, } \\
\text { exhibiendo un coeficiente } \\
\text { positivo y significativo } \\
\text { para todos los periodos } \\
\text { analizados. }\end{array}$ \\
\hline $\begin{array}{l}\text { García Borbolla y } \\
\text { Larrán Jorge (2005) }\end{array}$ & $\begin{array}{l}16170 \text { observaciones } \\
\text { de Alemania, Francia, } \\
\text { España, Holanda, } \\
\text { Italia, Suiza y Reino } \\
\text { Unido }\end{array}$ & $\begin{array}{l}\text { - Ratio de endeuda- } \\
\text { miento: fondos ajenos } \\
\text { / volumen de activo } \\
\text { total. } \\
\text { - Ratio de solvencia: } \\
\text { activo circulante / } \\
\text { pasivo circulante }\end{array}$ & $\begin{array}{l}\text { - Análisis descriptivo } \\
\text { - Test de Mann-Whit- } \\
\text { ney / Wilcoxon } \\
\text { - Regresión logística }\end{array}$ & $\begin{array}{l}\text { El grado de endeu- } \\
\text { damiento resultó } \\
\text { significativo sólo } \\
\text { en Alemania. La } \\
\text { liquidez, salvo en Reino } \\
\text { Unido, no incide en el } \\
\text { volumen de dividendos } \\
\text { repartidos }\end{array}$ \\
\hline
\end{tabular}

Fuente: Elaboración propia en base a la revisión bibliográfica 


\section{Metodología}

\subsection{Selección de la muestra}

A los fines de la estimación de la función clasificatoria, las empresas se consideraron según hayan distribuido dividendos (código 1) o no (código 0). Se trabajó con empresas de Argentina que cotizaron sus acciones en la Bolsa de Comercio de Buenos Aires entre los años 2003 y 2010. Esto permitió conformar una base de datos con los estados contables anuales de 70 empresas, lo que totalizó 533 observaciones. Se excluyeron algunas empresas que presentaban situaciones atípicas en cuanto a su actividad $\mathrm{y} / 0$ variables con valores nulos que impedían el cálculo de los ratios y podían distorsionar los resultados del análisis.

\subsection{Covariables}

En una primera etapa se seleccionó, del total de ratios incluidos en trabajos previos sobre la política de dividendos (Rodríguez Enríquez, 2003; García Borbolla y Larrán Jorge, 2005; Guzmán, 2004 y Rodríguez Fernández, 1988), nueve ratios. La Tabla No 3 presenta los ratios considerados inicialmente. También se incorporó el tiempo en el análisis, creando una variable numérica que se incrementa en una unidad por cada año transcurrido.

Tabla 3. Ratios contables seleccionados

\begin{tabular}{|c|c|}
\hline $\begin{array}{l}\text { RÓTULO DE } \\
\text { IDENTIFICACIÓN }\end{array}$ & RATIO \\
\hline$A C \_P C$ & $\begin{array}{l}\text { Activo corriente dividido por el pasivo } \\
\text { corriente }\end{array}$ \\
\hline PT_AT & Pasivo total dividido por el activo total \\
\hline V_AT & Ventas divididas por el activo total \\
\hline U_AT & $\begin{array}{l}\text { Utilidad antes de intereses e impuestos } \\
\text { dividida por el activo total }\end{array}$ \\
\hline R_RNA & $\begin{array}{l}\text { Reservas divididas por los resultados } \\
\text { no asignados }\end{array}$ \\
\hline FF_AT & $\begin{array}{l}\text { Flujo de fondos originado por las opera- } \\
\text { ciones dividido por el activo total }\end{array}$ \\
\hline E_AT & $\begin{array}{l}\text { Activos líquidos (o recursos de efectivo) } \\
\text { divididos por el activo total }\end{array}$ \\
\hline P_PN & Deudas divididas por el patrimonio neto \\
\hline CT_AT & $\begin{array}{l}\text { Capital de trabajo dividido sobre el activo } \\
\text { total }\end{array}$ \\
\hline
\end{tabular}

DEFINICIÓN

Medida de la solvencia y la liquidez general de la empresa.

Mide el endeudamiento de la empresa. Indica la rotación de los activos dentro de la empresa.

Mide la rentabilidad en función de las ganancias de la explotación.

Mide la financiación y endeudamiento de la empresa.

Medida de la posición del efectivo respecto del total de los recursos de la empresa.

Mide la proporción de los recursos más líquidos de la empresa sobre el total de sus recursos.

Medida del endeudamiento de la empresa.

Expresa el grado de fluidez de los activos e indica en qué medida la inmovilización del activo total resulta neutralizada por el capital de trabajo.

Fuente: Elaboración propia en base a la revisión bibliográfica

Con el objeto de explorar la existencia de diferencias significativas en los valores de los ratios contables según la condición de la empresa (reparte/no reparte dividendos), se aplicó, considerando cada año por separado, el Test $U$ de Mann-Whitney. Esta prueba, no paramétrica, resulta adecuada para comparar variables continuas en muestras independientes. Los resultados se muestran en la Tabla N ${ }^{0} 4$. 
Tabla 4. Resultados (valor p) del test de Mann-Whitney por año. Argentina

\begin{tabular}{|c|c|c|c|c|c|c|c|c|}
\hline \multirow[b]{2}{*}{ RATIO } & \multicolumn{8}{|c|}{ AÑo } \\
\hline & 2003 & 2004 & 2005 & 2006 & 2007 & 2008 & 2009 & 2010 \\
\hline AC_PC & $0,026^{*}$ & 0,172 & 0,146 & 0,616 & 0,429 & 0,115 & 0,731 & 0,718 \\
\hline PT_AT & $0,008^{*}$ & $0,002^{*}$ & 0,007 * & 0,359 & 0,275 & 0,101 & 0,310 & 0,204 \\
\hline V_AT & 0,435 & 0,899 & 0,294 & 0,533 & $\underline{0,373}$ & 0,198 & 0,705 & 0,173 \\
\hline U_AT & $0,009 *$ & 0,157 & $\underline{0,027^{*}}$ & 0,299 & 0,209 & $\underline{0,027 *}$ & 0,197 & $0,033^{*}$ \\
\hline R_RNA & $\underline{0,061^{*}}$ & $0,043^{*}$ & $\underline{0,051^{*}}$ & 0,168 & $0,068 *$ & $\underline{0,011^{*}}$ & 0,757 & $0,004^{*}$ \\
\hline FF_AT & $0,009 *$ & 0,418 & 0,379 & 0,959 & 0,240 & 0,860 & 0,630 & $0,005^{*}$ \\
\hline E_AT & 0,773 & 0,611 & 0,636 & 0,341 & 0,254 & 0,605 & 0,249 & 0,397 \\
\hline P_PN & $0,076^{*}$ & $0,015^{*}$ & $0,037^{*}$ & 0,678 & 0,469 & 0,377 & 0,692 & 0,562 \\
\hline CT_AT & $0,011^{*}$ & 0,187 & 0,108 & 0,640 & 0,314 & 0,241 & 0,642 & 0,766 \\
\hline
\end{tabular}

* Significativo a un nivel de 0,10. Fuente: Elaboración propia

\subsection{Modelos de respuesta binaria}

En este trabajo se aplicó un modelo donde la variable de respuesta es la variable binaria que indica el grupo al que pertenece la empresa (distribuye dividendos $=1$ y no distribuye $=0$ ) y las variables predictoras son los indicadores económico-financieros seleccionados. Los problemas con respuesta dicotómica pueden ser modelados a través de un modelo de regresión logística, donde el interés será la esperanza (media) de las respuestas, como función de las covariables. La esperanza de una respuesta binaria $\left(y_{i}\right)$, dada una covariable $\left(x_{i}\right)$, es la probabilidad de que la respuesta sea 1.

$E\left(y_{i} / x_{i}\right)=P\left(y_{i}=1 / x_{i}\right)=h\left(\beta_{1}+\beta_{2} x_{i}\right)$

Su transformación logit es:

$\operatorname{logit}\left\{P\left(y_{i}=1 / x_{i}\right)\right\} \equiv \ln \left\{\frac{P\left(y_{i}=1 / x_{i}\right)}{1-P\left(y_{i}=1 / x_{i}\right)}\right\}=$ $=\beta_{1}+\beta_{2} x_{i}$

En donde:

$\frac{P\left(y_{i}=1 / x_{i}\right)}{I-P\left(y_{i}=1 / x_{i}\right)}=\operatorname{Odds}\left(y_{i}=1 / x_{i}\right)$

Los odds representan las chances del éxito frente al fracaso y pueden expresarse alternativamente como:
$\operatorname{Odds}\left(y_{i}=1 / x_{i}\right)=\exp \left(\beta_{1}+\beta_{2} x_{i}\right)$

De esta manera, la probabilidad de que la respuesta sea 1 en el modelo logístico estará dada por:

$P\left(y_{i}=1 / x_{i}\right) \frac{\exp \left(\beta_{1}+\beta_{2} x_{i}\right)}{1+\exp \left(\beta_{1}+\beta_{2} x_{i}\right)}$

El modelo logístico clásico supone que las respuestas son independientes dadas las covariables, por lo que resulta apropiado cuando los datos no tienen ningún tipo de agrupamiento. Este requisito no se cumple en este estudio, ya que la estructura de los datos introduce dependencia en las respuestas múltiples dentro de cada unidad, aún condicionando sobre las covariables. Por esta razón, en este trabajo se utiliza un modelo logístico mixto, en el que la referida asociación puede ser modelada al incorporar un coeficiente aleatorio $\zeta_{j}$ en el predictor lineal (Rabe-Hesketh y Skrondal, 2012). Así, el modelo logístico con intercepto aleatorio $\zeta_{j}$ y una covariable $\left(x_{i j}\right)$ queda dado por:

$\operatorname{logit}\left\{P\left(y_{i j}=\frac{1}{x_{i j}}, \zeta_{i}\right)\right\}=\beta_{1}+\beta_{2} x_{i j}+\zeta_{j}$

Donde $i$ hace referencia al año y $j$ a la empresa. $\zeta_{i} \sim N(0, \psi)$ y se asumen independientes e idénticamente distribuidos entre empresas $j$ e indepen- 
dientes de las covariables $x_{i j}$. $\beta_{k}$ son los coeficientes de las covariables.

En función de los resultados del test no paramétrico se seleccionaron, para el ajuste inicial del modelo, aquellos ratios que resultaron estadísticamente significativos en al menos tres de los períodos analizados: PT_AT, U_AT, R_RNA y P_PN. Se realizaron varias corridas de prueba del modelo, permitiendo obtener un ajuste final con la incorporación de un coeficiente aleatorio específico por empresa $\zeta_{j}$ en el predictor lineal, que permitió capturar la asociación existente entre las respuestas de un mismo sujeto. Así se obtuvo un modelo logístico mixto, cuya formulación es la siguiente:

$$
\begin{aligned}
& \operatorname{logit}\left\{P\left(y_{i j}=1 / x_{i j}, \zeta_{j}\right)\right\}=\beta_{1}+\beta_{2} U \_A T_{i j}+ \\
& +\beta_{3} R \_R N A_{i j}+\beta_{4} P \_P N_{i j}+\beta_{5} \text { Tiempo o }_{i j}+\zeta_{j}
\end{aligned}
$$

En donde

$$
\begin{aligned}
& P\left(y_{i j}=1 / x_{i j}, \zeta_{j}\right)=\pi_{i j} \quad, y \\
& y_{i j} / \pi_{i j} \sim \operatorname{Binomial}\left(1, \pi_{i j}\right)
\end{aligned}
$$

Para ajustar este modelo se utilizó el software SAS con el procedimiento PROC GLIMMIX (modelo marginal 0 generalizado mixto), basado en linealizaciones, que no requiere valores iniciales de los parámetros. El método de estimación por defecto en PROC GLIMMIX para los modelos que contienen efectos aleatorios es una técnica conocida como cuasiverosimilitud penali- zada, RPL (Wolfinger y 0'Connell, 1993). Esta técnica emplea una expansión en torno a la estimación actual de los mejores predictores lineales insesgados de los efectos aleatorios, aunque suele producir estimaciones sesgadas de los parámetros de regresión en el caso de datos binarios con pocas repeticiones por sujeto. Para la selección del modelo más adecuado, dado el conjunto de datos disponibles, se evaluaron los criterios AIC, AICC y BIC provistos por PROC GLIMMIX para varios modelos alternativos. Finalmente se eligió el modelo que tuvo valores más pequeños para dichos criterios.

\section{Resultados}

Trabajando con un nivel de significación de 10 $\%$, los ratios R_RNA y P PN no resultaron significativos entre los predictores considerados para explicar contribuciones a las chances de que exista distribución de dividendos. Se advierte que el ratio de rentabilidad (U_AT) y el tiempo son significativos y el signo positivo de los coeficientes indica que aumentan las chances de que se repartan dividendos. En el caso del índice de rentabilidad, su odd ratio es de 8,7846 , es decir que por cada unidad que aumenta el ratio la chance de distribuir dividendos se incrementa en casi un $778 \%$ [100\%

\begin{tabular}{|c|c|c|c|c|}
\hline PARÁMETROS & ESTIMACIÓN & ERROR ESTÁNDAR & $P$ VALOR & $\begin{array}{l}\text { ODDS RATIOS VARIABLES } \\
\text { SIGIIFICATIVAS }\end{array}$ \\
\hline Intercepto & $-2,4394$ & 0,3695 & $<0,0001^{*}$ & \\
\hline Tiempo & 0,1835 & 0,05768 & $0,0016^{*}$ & 1,2014 \\
\hline U_AT & 2,1730 & 1,1218 & 0,0534 * & 8,7846 \\
\hline R_RNA & $-0,00615$ & 0,01902 & 0,7466 & \\
\hline P_PN & $-0,08028$ & 0,07709 & 0,2983 & \\
\hline Varianza intercepto aleatorio & 2,6415 & 0,6776 & 0,0001 * & \\
\hline
\end{tabular}
(odds-1)]. Respecto del tiempo, la chance de repartir dividendos aumenta en un $20 \%$ por año.

Tabla 5. Estimaciones de los parámetros del modelo logístico con intercepto aleatorio

* Significativo a un nivel de 0,10. Fuente: Elaboración propia 
El objetivo principal en este tipo de estudios es realizar inferencias acerca de los efectos fijos relacionados a promedios poblacionales. La inclusión de efectos aleatorios es un mecanismo para caracterizar la correlación entre las mediciones para un mismo sujeto, pero en general no interesan conclusiones sujeto-específico. Sin embargo, la estimación de la desviación estándar de la ordenada aleatoria del modelo constituye un resumen útil del grado de heterogeneidad de la población bajo estudio (Agresti, 2002). En la aplicación abordada en este trabajo la varianza de la ordenada al origen aleatoria $(\psi=2,6415)$ es significativamente distinta de cero ( $p=0,0001)$, lo que sugiere que la heterogeneidad en la población es importante, siendo atribuible la misma a diferencias entre las empresas.

\section{Conclusiones}

Numerosos trabajos sobre la decisión de las empresas de distribuir dividendos coinciden en la utilización del análisis de regresión para la estimación del poder explicativo de diferentes variables y otros aplican técnicas multivariadas, como el análisis de componentes principales 0 la regresión logística. El modelo logístico permite levantar el supuesto de normalidad de los datos. No obstante, un supuesto clave que se mantiene es la independencia entre las observaciones, lo que afecta la variabilidad de las estimaciones. Por otro lado, varias mediciones de las mismas unidades permiten captar su heterogeneidad no observable induciendo correlación entre las respuestas. Es necesario, entonces, trabajar con modelos que incorporen esa correlación. Esto puede realizarse desde un enfoque promedio poblacional (modelo marginal) 0 desde los modelos de efectos aleatorios, también denominados mixtos, en los que la asociación puede ser modelada incluyendo ordenada al origen 0 coeficientes aleatorios (Rabe-Hesketh y Skrondal, 2012).

Dado que en este trabajo el interés estuvo centrado en una respuesta binaria (reparte / no reparte dividendos), se utilizaron modelos genera- lizados para respuesta dicotómica. Para contemplar la correlación en las series de datos longitudinales se utilizaron extensiones de estos modelos lineales generalizados para datos binarios (Fahrmeir y Tutz, 2001). El uso del enlace logit fue preferido debido a que la interpretación de los parámetros en términos de cocientes de chances resultó apropiada para evaluar el efecto de los factores de interés sobre la decisión de repartir dividendos. Se ajustó un modelo logístico con ordenada al origen aleatoria para medidas repetidas binarias, que incorpora vía el coeficiente aleatorio la correlación entre las respuestas de una misma empresa, al tiempo que permite modelar la dependencia de la respuesta en términos de las variables explicatorias. Ignorar la correlación entre las respuestas podría provocar que se detecten erróneamente efectos no significativos de las variables predictoras, ya que los errores estándares son subestimados.

El modelo ajustado permitió identificar como variable con alta capacidad discriminadora al ratio de rentabilidad (U_AT), lo que incrementa significativamente la probabilidad de que la empresa distribuya dividendos. También resultó significativo el paso del tiempo, que incrementa la chance de repartir dividendos un $20 \%$ por año.

De esta manera, y en consonancia con los resultados hallados por la mayor parte de las investigaciones, mayor es la propensión a distribuir dividendos cuanto más rentable es la empresa. La causa parece más que obvia, sin utilidades no hay dividendos que repartir. La generación de rentas coloca a la empresa en condiciones de remunerar a sus propietarios repartiendo dividendos (Rodríguez Fernández, 1988). Este resultado se contrapone con la hipótesis de que las empresas rentables podrían retener dividendos como señal para los accionistas de capacidad de reinversión y obtención de mayores ganancias de capital futuras. La evidencia en este caso parece mostrar que los accionistas revelan mayor preferencia por ganancias actuales que por ganancias futuras. Esto coincide con lo expuesto por Rodríguez Enríquez (2003), quien a su vez cita 
los trabajos de Azofra Palenzuela (1986) y Fama y French (2001), y sostiene que la rentabilidad es uno de los factores que mayor influencia puede ejercer sobre la política de reparto de dividendos, pues cuanto más elevados son los niveles de rentabilidad, mayor es la capacidad de las empresas para distribuir dividendos. Siguiendo un razonamiento similar, García Borbolla y Larrán Jorge (2005) suponen que esta relación positiva entre las utilidades y la distribución de dividendos encuentra causa en que los mayores beneficios permiten mayor disponibilidad de fondos para usos alternativos por parte de las empresas, constituyendo el reparto de dividendos una de estas opciones.

En cuanto a la influencia positiva del paso del tiempo sobre las chances de repartir dividendos, una posible explicación es la ofrecida por Rodríguez Fernández (1988), quien supone probable que la madurez de las firmas tiende a proporcionar dividendos elevados y constantes, mientras que las empresas en fase de crecimiento suelen acumular todas 0 gran parte de sus utilidades.

\section{Referencias bibliográficas}

- Agresti, A. (2002). Categorical Data Analysis (2nd.). New York: John Wiley and Sons.

- Asadi, G.H., y Azizi Basir, S. (2008). Studying the relationship between profitability and liquidity of the Company and its impact on the profit dividend. Quarterly of Management Message, 28, 133-155.

- Azofra Palenzuela, V. (1986). Las decisiones de distribución de dividendos en la gran empresa española: Sus variables determinantes. Anales de Estudios Económicos y Empresariales, 1, 257-274.

- Black, F. (1976). The dividend puzzle, Journal of Portfolio Management, 2(2), 5-8.

- Darling, P. (1957). The influence of expectations and liquidity on dividend policy. Journal of Political Economy, 65(3), 209-224.

- Dhrymes, P.J. y Kurz M. (1964). On dividend policy of electric utilities. Review of Economics and Statistics, 46(1), 76-81.
- Diggle, P., Heagerty, P., Liang, K.Y, y Zeger, S.L. (2002). Analysis of Longitudinal Data (2nd Ed.). New York: Oxford University Press.

- Fahrmeir, L. y Tutz, G.T. (2001). Multivariate Statistical Modelling Based on Generalized Linear Models (2nd Edition). New York: Springer-Verlag.

- Fama, E.F., y Babiak, H. (1968). Dividend policity: an empirical analysis. Journal of the American statistical Association, 63(324), 1132-1161.

- Fama, E.F., y French, K.R. (2001). Disappearing Dividends: Changing Firm Characteristics or Lower Propensity to Pay. Journal of Financial Economics, 60(1), 3-43.

- Friend, I., y Puckett, M. (1964). Dividends and Stock Prices. The American Economic Review, 54(5), 656-682.

- García Borbolla, A., y Larrán Jorge, M. (2005). Factores explicativos de la política de dividendos: evidencias empíricas en mercados bursátiles europeos. Boletín de estudios económicos, 60(185), 209-227.
- Gordon, M.J. (1962). The savings, investment and valuation of a corporation. Review of Economics and Statistics, 45(1), 37-51.

- Guzmán, I. (2004). Factores explicativos del reparto de dividendos a cuenta en empresas españolas. Cartagena, España: Instituto Valenciano de Investigaciones Económicas. - Jensen, M.C., y Smith, C.W. (1984). The modern theory of corporate finance. New York: McGraw-Hill Series in Finance. - Kanwer, A. (2003, Abril). The determinants corporate dividend polices in Pakistan. Pakistan and Gulf Economist. Recuperado de http:// www. pakistaneconomist.com/issue2003/issue17/f\&m4.php

- Lintner, J. (1956). Distribution of income of corporations among dividends, retained earnings, and taxes. American Economic Review, 46(2), 97-113.

- Miller, M.H., y Modigliani, F. (1961). Dividend policy, growth, and the valuation of shares. Journal of Business, 34(4), 411-433. 
- Rabe-Hesketh, S., y Skrondal, A. (2012). Multilevel and Longitudinal modeling using Stata, (3rd ed.). CollegeStation, Texas: Stata Press Publication.

- Rodríguez Enríquez, E. (2003). Caracterización económica-financiera de las Pymes en función de la decisión de reparto de dividendos. Revista española de Financiación y Contabilidad, XXXII(119), 1137-1175. - (1988). La decisión de
distribuir dividendos: investigación empírica en la gran empresa española. Investigaciones Económicas, XII(2), 243-258.

- Suárez Suárez, A.S. (1986). Decisiones óptimas de inversión y financiación en la empresa (8va. Ed.). Madrid: Pirámide.

- Tabari, N., y Shirazi, S. (2015). Examining the determinants of dividend policy in listed companies in Theran Stock Exchange. Indian Journal of Fundamental and Applied Life Sciences, 5(3), 2375-2382.
- Truong, T., y Heaney, R. (2002). Largest shareholder and dividend policy around the world. The Quarterly Review of Economics and Finance, 47, 667-687.

- Wolfinger, R., y O'Connell, M. (1993). Generalized linear mixed models: a pseudo-likelihood approach. Journal of Statistical Computation and Simulation, 48, 233-43.

\section{Registro bibliográfico}

González, M.V., y Moneta Pizarro, A.M. (2016). Predicción de la distribución de dividendos en Argentina empleando un modelo lineal generalizado mixto. Revista Ciencias Económicas, 13(02), 9-20. 